\title{
Criminologie
}

\section{L'évaluation de la validité prédictive du LS/CMI dans la prédiction des incidents disciplinaires en prison}

\section{Thibault Charton, Jean-François Couture-Poulin et Jean-Pierre Guay}

Volume 44, numéro 2, automne 2011

URI : https://id.erudit.org/iderudit/1005800ar

DOI : https://doi.org/10.7202/1005800ar

Aller au sommaire du numéro

Éditeur(s)

Les Presses de l’Université de Montréal

ISSN

0316-0041 (imprimé)

1492-1367 (numérique)

Découvrir la revue

Citer cet article

Charton, T., Couture-Poulin, J.-F. \& Guay, J.-P. (2011). L'évaluation de la validité prédictive du LS/CMI dans la prédiction des incidents disciplinaires en prison. Criminologie, 44(2), 279-303. https://doi.org/10.7202/1005800ar
Résumé de l'article

Dans son plan stratégique, le ministère de la Sécurité publique du Québec souligne les problèmes actuels de surpopulation carcérale au Québec. Ce phénomène peut contribuer à une augmentation des conflits vécus par les détenus et, par extension, à l'émergence des incidents disciplinaires. Dans un tel contexte, il semble nécessaire d'améliorer la gestion du risque lié à ce type d'incidents. Dans le cadre de cet article, l'objectif principal visé est de mettre à l'épreuve la validité prédictive du LS/CMI sur l'occurrence des incidents disciplinaires. L'échantillon consiste en 934 détenus incarcérés au Service correctionnel du Québec (SCQ) entre janvier et décembre 2007. Durant cette même période, tous les détenus ont été évalués dans les semaines suivant leur admission avec la version québécoise du LS/CMI. Les résultats permettent de confirmer l'efficacité du LS/CMI dans la prédiction des incidents disciplinaires, et ce, indépendamment de leur nature et de leur cible. 


\title{
L'évaluation de la validité prédictive du LS/CMI dans la prédiction des incidents disciplinaires en prison
}

\author{
Thibault Charton \\ Centre international de criminologie comparée (CICC) \\ École de criminologie, Université de Montréal \\ thibaultcharton@umontreal.ca \\ Jean-François Couture-Poulin \\ Direction de la recherche, ministère de la Sécurité publique du Québec \\ jean-francois.couture-poulin@msp.gouv.qc.ca \\ Jean-Pierre Guay \\ Centre international de criminologie comparée (CICC) \\ École de criminologie, Université de Montréal \\ Institut Philippe-Pinel de Montréal \\ jean-pierre.guay@umontreal.ca
}

RÉSUMÉ - Dans son plan stratégique, le ministère de la Sécurité publique du Québec souligne les problèmes actuels de surpopulation carcérale au Québec. Ce phénomène peut contribuer à une augmentation des conflits vécus par les détenus et, par extension, à l'émergence des incidents disciplinaires. Dans un tel contexte, il semble nécessaire d'améliorer la gestion du risque lié à ce type d'incidents. Dans le cadre de cet article, l'objectif principal visé est de mettre à l'épreuve la validité prédictive du LS/CMI sur l'occurrence des incidents disciplinaires. L'échantillon consiste en 934 détenus incarcérés au Service correctionnel du Québec (SCQ) entre janvier et décembre 2007. Durant cette même période, tous les détenus ont été évalués dans les semaines suivant leur admission avec la version québécoise du LS/CMI. Les résultats permettent de confirmer l'efficacité du LS/CMI dans la prédiction des incidents disciplinaires, et ce, indépendamment de leur nature et de leur cible.

MOTS-CLÉS - Incidents disciplinaires, prison, gestion du risque, instrument actuariel. 


\section{Introduction $^{1}$}

Bien que la question de la prise en charge des délinquants en soit une qui est particulièrement délicate, les prisons sont désormais plus difficiles à gérer (Dilulio, 1991; Gendreau et al., 1997). L'une des principales causes de cette difficulté est vraisemblablement le phénomène de surpopulation carcérale, lequel a atteint un sommet vers la fin des années 1990 au Québec (Landreville et Charest, 2004). Le taux d'occupation moyen des établissements de détention au Québec avoisine $100 \%$ depuis le début des années 1990, variant de 98,0\% pour l'année 1999-2000 à 114,6\% pour l'année 1994-1995 (Landreville et Charest, 2004). Il semble que la situation ne s'est guère améliorée récemment. En 2009, il y avait 4705 détenus dans les établissements de détention du Québec alors que la capacité d'accueil était de 3900 places (Assemblée nationale du Québec, 2009). Afin de répondre à cette situation, les administrateurs carcéraux ont procédé à des modifications de l'environnement physique. Les cellules ont été converties afin d'accueillir jusqu'à trois détenus, et bien souvent les salles communes ont été transformées en dortoirs. Par exemple, Robinson et Mirabelli (1996) constatent que 26,4\% de leur échantillon, composé de 4285 détenus canadiens, ont déclaré qu'ils partageaient leur cellule avec au moins un autre détenu. Plus la population carcérale a tendance à augmenter, plus les conditions de vie à l'intérieur des prisons se dégradent et le climat de tension s'intensifie. Dans ces conditions, le nombre d'incidents graves de sécurité, comprenant le meurtre, les évasions, la séquestration et la prise d'otages, est passé de 86 à 109, et le nombre de voies de fait, de 756 à 911 dans les établissements de détention du Québec entre 2004 et 2007 (Services correctionnels du Canada, 2007). À cet égard, le contrôle des détenus en milieu carcéral est essentiel afin de réduire les coûts humains et économiques associés à la commission d'incidents disciplinaires (Goetting et Howsen, 1986; Lovell et Jemelka, 1996).

D'un point de vue économique, les frais médicaux, administratifs et sécuritaires (transfert, isolement) relatifs à la réponse aux incidents disciplinaires atteignent des sommes substantielles, payées par l'État et les contribuables. Il y a déjà plus de 10 ans, Lovell et Jemelka (1996) estimaient que le traitement d'un incident disciplinaire violent revenait à environ 1000 \$ par incident aux États-Unis. D’un point de vue

1. Les opinions exprimées n'engagent que les auteurs et ne sont pas nécessairement celles du ministère de la Sécurité publique du Québec. 
humain, les incidents disciplinaires peuvent nuire au bien-être et à la santé des détenus et des membres du personnel carcéral (Goetting et Howsen, 1986; Wooldredge, 1991; O'Donnell et Edgar, 1998). Ces incidents entraînent des conséquences pouvant aller du stress lié au séjour dans un milieu instable aux blessures physiques nécessitant une intervention médicale. Ce sont ces implications, du moins en partie, qui ont poussé plusieurs chercheurs à s'intéresser à la question des incidents disciplinaires et aux facteurs qui y sont associés.

\section{La mesure du risque d'incidents disciplinaires}

Lapplication pratique des notions acquises par la mise à l'épreuve des modèles théoriques est souvent la création d'un instrument permettant d'identifier les délinquants les plus à risque de faire l'objet de rapports $\mathrm{d}$ 'incidents disciplinaires. Une telle application permet, entre autres, de faciliter le classement sécuritaire et la répartition des énergies relatives à la surveillance (ex.: nombre de gardiens). Les instruments (spécialisé et génériques) présentés dans la sous-section suivante ont fréquemment été utilisés dans le cadre de l'évaluation du niveau de risque sécuritaire et la prédiction des incidents disciplinaires.

\section{Le Risk Assessment Scale for Prison}

Le Risk Assessment Scale for Prison (RASP) de Cunningham et al. (2005) est un instrument actuariel qui permet de mesurer le niveau de risque d'incidents disciplinaires violents dans les prisons à sécurité maximale. Cet instrument fut construit à l'aide d'un échantillon de 1503 détenus admissibles à une libération conditionnelle, de 960 détenus condamnés à une peine d'emprisonnement à perpétuité et de 132 détenus condamnés à mort. Ces détenus étaient incarcérés entre 1991 et 2002 au Centre correctionnel Potosi au Missouri. Les prédicteurs comprennent des facteurs tels que l'âge, le niveau d'éducation, les antécédents criminels, le type de crime et la durée de la sentence. Les incidents disciplinaires violents comprennent le meurtre, les tentatives de meurtre, le viol ainsi que les agressions majeures et mineures accumulées sur une période de 11 ans.

Les résultats indiquent que l'âge est le prédicteur le plus puissant. Les détenus âgés de moins de 21 ans $(\beta=1,16, p<0,001)$ commettent un plus grand nombre d'incidents violents pendant leur incarcération. 
Par ailleurs, le niveau de scolarité $(\beta=-0,573, p<0,001)$, les antécédents criminels $(\beta=0,341, p<0,01)$ et les crimes contre la propriété $(\beta=0,432, p<0,001)$ permettent de prédire significativement les incidents disciplinaires violents. Dans l'ensemble, le RASP donne une aire sous la courbe (AUC) ROC de 0,72, une prédiction qualifiée de modeste (Swets, 1988). La révision de cet instrument (Cunningham et Sorensen, 2006) avec un échantillon plus important de détenus $(\mathrm{N}=13$ 341) et l'ajout de certains facteurs n'a cependant pas permis de voir augmenter la valeur du coefficient d'aire sous la courbe (AUC $=0,65$ à 0,71 ). Bien que le RASP soit une des seules initiatives visant à concevoir un instrument destiné à évaluer le risque sécuritaire, il comporte certaines limites. En effet, il ne prend en compte que des facteurs issus du contexte et de l'environnement carcéral, comme la surpopulation. Un tel choix pourrait d'ailleurs expliquer en partie le fait que les modèles ne permettent d'atteindre que difficilement le seuil de 0,70 . À ce sujet, une part des difficultés à prédire les incidents disciplinaires est possiblement due au fait que la variable dépendante ne différencie pas la planification, le complot et la tentative d'acte violent.

Puisque les prédicteurs associés sont relativement similaires aux facteurs de risque utiles afin de prédire la récidive criminelle générale, et que l'implantation d'un outil propre à la gestion du risque sécuritaire est onéreuse, certains chercheurs se sont penchés sur l'utilité d'instruments génériques pour prédire le risque d'incidents disciplinaires.

\section{Les outils génériques}

Le nombre d'échelles et d'instruments génériques utilisés pour prédire les incidents disciplinaires est relativement élevé. Cependant, quatre d'entre eux ont fait l'objet d'une attention plus particulière : la PCL-R, le VRAG, le MMPI et le LSI-R.

\section{La PCL-R}

L'échelle de psychopathie de Hare (PCL-R, 2003) est une échelle qui permet d'évaluer les traits de la personnalité psychopathique. Elle contient 20 items qui sont codés sur une échelle de trois points $(0=$ absent ; 1 = possibilité ou partiellement présent; 2 = présent). Le total varie de 0 à 40 . Elle est devenue rapidement l'outil par excellence pour mesurer le niveau de psychopathie chez les délinquants. En ce qui a 
trait à la prédiction des incidents disciplinaires par la PCL-R, les résultats sont mitigés (Edens et al., 1998). Bien que plusieurs chercheurs aient été en mesure d'établir une relation significative entre la psychopathie et les incidents disciplinaires violents (Serin, 1991; Edens et al., 1999), d'autres (Young et al., 2004; Endrass et al., sous presse) n'ont pas été en mesure d'observer une telle relation. Endrass et ses collègues (sous presse) ont étudié le lien éventuel entre la psychopathie et les incidents disciplinaires auprès d'un échantillon de 113 détenus condamnés pour des crimes violents ou sexuels. Les données concernant les incidents disciplinaires provenaient des dossiers carcéraux officiels. Ils ont constaté une association non significative entre le facteur 1 , le facteur 2, le résultat total à la PCL-R et les incidents disciplinaires impliquant de la violence physique. Néanmoins, leurs résultats indiquent une prédiction modeste des incidents impliquant de la violence verbale par le facteur $1(\mathrm{AUC}=0,70)$ et le résultat total à la PCL-R $(A U C=0,69)$. Pour leur part, Walters, Duncan et Geyer (2003) ont suivi 185 détenus fédéraux pendant deux ans. Avec une définition plus large des incidents disciplinaires (incluant les incidents d'agressions physiques et verbales), ils ont observé seulement une modeste association statistique avec un $r$ de 0,11 et un AUC de 0,58.

\section{Le VRAG}

Le Violence Risk Appraisal Guide (Harris et al., 1993) fut conçu afin de prédire la récidive violente. Il fut initialement validé avec un échantillon de 618 hommes suivis entre 7 et 10 ans dans un hôpital à sécurité maximale. Il fut également validé avec des détenus non psychiatrisés (Loza et Dhaliwa, 1997). Cet instrument contient des variables démographiques, psychologiques et de carrière criminelle. Kroner et Mills (2001) ont testé la validité prédictive du VRAG en lien avec les incidents disciplinaires. Leur étude portait sur un suivi de 8 mois de 98 détenus canadiens. Les incidents disciplinaires étaient regroupés selon leur sévérité, soit les incidents majeurs (émeutes, menaces, utilisation de drogues et d'alcool, assauts, refus d'obtempérer à un ordre direct) et les incidents mineurs (tenue vestimentaire inappropriée, non-respect d'un officier et des directives). Les résultats ont permis de révéler une relation modérée entre les incidents mineurs $(r=0,40)$, majeurs $(r=0,26)$ et les scores au VRAG (duquel on a retiré l'item de la PCL-R). Les analyses de courbe ROC indiquent une prédiction modeste à modérée avec 
des indices AUC de 0,76 pour les incidents mineurs et de 0,63 pour les incidents majeurs.

\section{Le MMPI}

Le Minnesota Multiphasic Personality Inventory (Butcher et al., 1989) est un questionnaire d'autoévaluation qui vise à étudier la psychopathologie. Cet inventaire contient trois échelles d'attitude et 10 échelles de personnalité pathologique: Hypocondrie, Dépression, Hystérie, Personnalité psychopathique, Masculinité-féminité, Paranoïa, Psychasthénie, Schizophrénie, Hypomanie et Introversion sociale. Grâce à sa simplicité d'administration, le MMPI est largement utilisé dans le milieu carcéral et psychiatrique. La méta-analyse de Gendreau et ses collaborateurs (1997) indique que l'association entre les échelles du MMPI et les incidents disciplinaires est pour le moins modeste, comme en témoigne une corrélation de 0,07.

\section{Le LSI-R}

Le Level of Service Inventory - Revised est un instrument actuariel qui permet d'évaluer les besoins et les facteurs de risque du contrevenant afin de faciliter les décisions des intervenants quant à son traitement et à son niveau de supervision (Andrews et Bonta, 1995). Il contient 54 items statiques et dynamiques compris dans 10 dimensions: histoire criminelle, éducation et emploi, famille et état civil, loisirs et passetemps, relations sociales, abus d'alcool et de drogues, attitudes procriminelles, patterns antisociaux, adaptation et situation financière. Depuis sa création, cet instrument a prouvé sa fiabilité dans la prédiction du risque de récidive (Hollin et Palmer, 2006). Le LSI-R semble surpasser l'ensemble des autres outils actuariels et les inventaires de personnalité (Gendreau et al., 1997) dans la prédiction des incidents disciplinaires. Généralement, la corrélation entre le LSI-R et les incidents disciplinaires se situe entre 0,26 à 0,39 selon les travaux de Bonta (1989) et Bonta et Montiuk (1987).

Dans la même veine, Kroner et Mills (2001) ont comparé auprès d'un échantillon de 97 détenus la validité prédictive de cinq instruments actuariels génériques (la PCL-R, le LSI-R, la HCR-20 de Webster, Eaves, Douglas, Wintrup [1995], le VRAG et le Lifestyle Criminality Screening Form [LCSF] de Walters, White et Denney [1991]) par rapport aux incidents disciplinaires. Pour la prédiction des incidents discipli- 
naires mineurs, le VRAG obtient l'indice AUC le plus élevé (AUC = $0,76)$, tandis que le LCSF obtient le plus faible $(0,61)$. Les trois autres instruments obtiennent des indices d'aire sous la courbe ROC de 0,68 pour la HCR-20, de 0,66 pour le LSI-R et la PCL-R. Les résultats d'aire sous la courbe ROC pour les incidents disciplinaires majeurs étaient généralement plus faibles que les résultats des incidents mineurs. Les deux seuls instruments franchissant la barre des 0,60 sont le VRAG et le LSI-R avec des indices AUC de 0,63 et 0,61 respectivement. Ces résultats indiquent que le LSI-R et le VRAG semblent être les instruments les plus précis dans la prédiction des incidents disciplinaires contre la personne. Par contre, aucun instrument ne s'est réellement démarqué. La grande similitude entre les items de chacun des instruments peut en partie expliquer de tels résultats.

La prédiction des incidents disciplinaires semble être un champ d'études florissant, tel qu'en témoigne un nombre croissant de publications. Néanmoins, elle demeure une entreprise complexe, et l'état actuel des travaux suggère un nombre important de limites. Parmi les plus importantes, on trouve l'utilisation d'échantillons restreints (par ex. : Kroner et Mills, 2001; Zamble, 1992), parfois même comportant des biais de sélection importants (par ex. : Thomas et Foster, 1973; Paterline et Petersen, 1999; Jiang et Fischer-Giorlando, 2002), une mesure peu détaillée des incidents disciplinaires (par ex.: Van Voorhis, 1994; Woolredge et al., 2001; Walters, 2003; Hochstetler et DeLisi, 2005), voire une confusion dans la séquence temporelle des événements (par ex.: Wright, 1991).

Le présent article vise deux objectifs distincts mais liés. Dans un premier temps, nous testons la validité prédictive du LS/CMI en lien avec les incidents disciplinaires selon leur sévérité et leur cible. Dans un deuxième temps, nous évaluons la contribution de certains facteurs incontournables dans la prédiction des incidents disciplinaires. Des facteurs tels que la surpopulation (Megargee, 1976; Gaes et McGuire, 1985; Rubback et Carr 1993; Gendreau et al., 1997; Wooldredge et al., 2001; Reisig, 2002; Tartaro, 2002), l'âge du détenu (Flanagan, 1983; Goetting et Howsen, 1986; Mackenzie, 1987; Cooper et Werner, 1990; Cao et al., 1997; Gendreau et al., 1997; Wooldredge et al., 2001; Cunningham et Sorensen, 2007) et son affiliation au crime organisé (Shelden, 1991; Gaes et al., 2002; DeLisi et al., 2004; Griffin et Hepburn, 2006; Cunningham et Sorensen, 2007; Trulson, 2007) ont démontré dans les études passées une influence significative sur la commission d'incidents disciplinaires en milieu carcéral. 


\section{Méthodologie}

\section{Participants}

L'échantillon consistait en 934 détenus incarcérés au Service correctionnel du Québec (SCQ) entre janvier 2007 et décembre 2007 (le SCQ prend en charge tous les délinquants ayant fait l'objet d'une peine correctionnelle d'au moins deux ans moins un jour). Chacun devait répondre à l'une ou l'autre des trois conditions suivantes: être condamné à une peine correctionnelle de plus de six mois, être condamné pour un crime violent ou avoir un risque dit documenté (par ex. : être lié au crime organisé). Aucun prévenu n'a été retenu dans cet échantillon en raison de l'accessibilité aux données et aux résultats des études précédentes sur le sujet (Ouimet, 1993). Tous les détenus ont été évalués dans les semaines suivant leur admission avec la version québécoise du LS/ CMI (Andrews et al., 2004) durant cette même période. Les participants étaient tous des hommes âgés entre 17 et 80 ans (moyenne $=36$ ) et provenaient des 18 établissements de détention du Québec.

\section{Instrument}

Le LS/CMI est un instrument qui permet d'évaluer et de gérer le risque que posent les délinquants (Andrews et al., 2004). Il est principalement basé sur l'approche de la personnalité générale et la théorie de l'apprentissage social d'Andrews et Bonta (2006). Il aide les intervenants à planifier le traitement et la gestion générale du contrevenant tout en évaluant son risque de récidive. Il s'appuie principalement sur une entrevue avec le contrevenant et l'étude approfondie de son dossier. Pour corroborer les informations recueillies, le rapport de police et d'autres données pertinentes doivent être utilisés. Bien que le LS/CMI soit disponible en version informatisée, la version papier a été employée dans le cadre de la présente recherche, et les données ont fait l'objet d'une saisie validée par un technicien préalablement formé.

Les travaux sur le LS/CMI indiquent qu'il est aussi performant pour une grande variété de populations, notamment les femmes délinquantes (Coulson et al., 1996; Rettinger, 1998; Girard, 1999), les délinquants autochtones (Bonta, 1989; Andrews et al., 2001), les jeunes délinquants de sexe masculin et féminin (Sparring, 1982; Shields et Simourd, 1991 ; Shields, 1993 ; Hoge et al., 1995 ; Andrews et al., 2001 ; Nowicka-Sroga, 2003) et les délinquants aux prises avec des problèmes de santé mentale (Andrews et al., 2001 ; Girard et Wormith, 2004). 
Le LS/CMI est un instrument de quatrième génération et comprend 11 sections. Seule la première section, laquelle permet de calculer la cote de risque, a été utilisée. Elle comprend 8 sous-sections : antécédents criminels (8 items), éducation ou emploi (9 items), famille ou couple (4 items), loisirs et activités récréatives ( 2 items), fréquentations (4 items), problèmes d'alcool ou de drogues ( 8 items), attitudes procriminelles (4 items) et comportements antisociaux (4 items). Cette section contient 43 items, raffinant les 54 items du LSI-R. Le risque évalué à l'aide du LS/CMI correspond à cinq niveaux, soit Très faible (0-4), Faible (5-10), Moyen (11-19), Élevé (20-29) et Très élevé (30-43). Plus le niveau de risque est élevé, plus le risque est élevé, et plus les besoins en matière d'intervention sont grands.

\section{Mesure des prédicteurs}

Les données du présent article proviennent essentiellement de deux sources. Comme première source, les échelles et le total au LS/CMI ont été utilisés.

Les données de la seconde source proviennent du système du dossier administratif correctionnel, le DACOR. Cette base de données permet aux intervenants du Service correctionnel du Québec et aux intervenants de la Commission des libérations conditionnelles de consigner des informations et d'avoir accès aux informations complètes du dossier correctionnel $\mathrm{du}$ contrevenant pendant tout le processus judiciaire. Cette base de données contient, entre autres, des informations sur l'assignation des cellules, le suivi des déplacements externes et l'ensemble des comportements problématiques du contrevenant pendant tout le processus judiciaire.

DACOR a permis d'accumuler des données supplémentaires sur le contrevenant depuis son incarcération. Les facteurs extraits de cette base sont l'âge du détenu et son affiliation à un gang. Le facteur de l'affiliation à un gang est également une échelle dichotomique. L'information était codifiée par un agent de renseignements spécifiquement formé à cette fin et en lien constant avec les services de police.

DACOR a également permis de recueillir des données relatives au taux d'occupation carcérale. La mesure du taux d'occupation carcérale a été notée à partir de la capacité réelle et de la capacité disponible associées aux différents établissements de détention durant chaque mois de l'année 2007. Ce facteur représente un pourcentage d'occupation 
(capacité réelle/capacité disponible) relatif au mois précédent la date de perpétration d'un incident par le détenu. Par conséquent, chaque détenu a son propre score de surpopulation, lequel est fonction du moment de sa présence et de l'établissement.

\section{Mesure des incidents disciplinaires}

Les informations sur les incidents disciplinaires ont été extraites du système DACOR. Ces incidents s'étaient déroulés entre février et décembre 2007. Ils ont été regroupés selon leur nature et leur cible, soit les incidents non violents, de violence physique, de violence verbale, visant un intervenant ou un détenu. La variable des incidents de violence verbale tenait compte des comportements comme l'utilisation de menaces, d'un langage ou de gestes inappropriés. La variable des incidents non violents, quant à elle, était définie par le dommage causé à des biens, le refus de participer et l'entrave aux tâches carcérales quotidiennes. Ce grand éventail d'incidents disciplinaires permet d'évaluer le risque d'incidents disciplinaires de différentes nature et sévérité.

\section{Analyses statistiques}

Afin d'évaluer la validité prédictive du LS/CMI et de ses sous-échelles, nous avons eu recours aux analyses de courbes ROC (Receiver Operating Characteristic). Les analyses de courbes ROC ont pour principal avantage d'être très peu sensibles au faible taux de base (Rice et Harris, 1995) généralement observable dans les travaux sur la délinquance. Les analyses de courbes ROC permettent de connaitre la sensibilité et la spécificité des modèles de privation et de l'importation, et ainsi, d'en connaitre la validité prédictive. Elle est donc la représentation graphique de la proportion des vrais positifs (sensibilité) par rapport à la proportion de faux positifs (1-spécificité) d'un test en fonction du changement de point de coupure. Le coefficient généralement utilisé pour faire état de la qualité de la prédiction est l'indice d'aire sous la courbe ROC. Cet indice varie entre 0 et 1 , où 0,5 correspond au hasard et 1 , à une prévision parfaite. Cet indice peut être généralement considéré comme la probabilité qu'un récidiviste choisi au hasard ait une cote plus élevée qu'un non-récidiviste également choisi au hasard (Hanson et Thornton, 1999). La qualité de l'indice AUC peut être évaluée à l'aide de l'échelle suivante: faible (0 à 
$0,60)$, marginale $(0,60$ à 0,70$)$, modeste $(0,70$ à 0,80$)$, modérée $(0,80$ à $0,90)$ et élevée (0,90 à 1) (Sjostedt et Grann, 2002).

\section{Résultats}

\section{Description des résultats sommaires}

Notre échantillon contient 88 détenus ayant commis un incident de violence physique; 100 ayant commis un incident de violence verbale; 141, un incident non violent; 121 , un incident visant un intervenant; ainsi que 308, visant un détenu. Durant la période d'étude, seulement quatre détenus avaient participé à plus de dix incidents disciplinaires. Un total de 938 incidents disciplinaires a été rapporté durant l'année 2007. Par ailleurs, la cooccurrence des divers types d'incidents a été étudiée grâce à des analyses de corrélation. Les résultats suggèrent une relation significative et positive entre l'ensemble des types d'incidents disciplinaires $(p<0,01)$. L'association entre les incidents de violence verbale et ceux impliquant un agent de services correctionnels, ainsi que les incidents non violents et ceux impliquant un codétenu sont les plus fortement corrélés avec un $r$ de Pearson de 0,77 et 0,66 respectivement. Finalement, le lien entre les incidents disciplinaires selon leur degré de gravité est faible, variant de 0,32 pour les incidents de violence physique et les non violents à 0,41 pour les incidents de violence verbale et les non violents.

Les statistiques descriptives de notre échantillon pour l'ensemble des variables contrôles utilisées, ainsi que pour les huit échelles du LS/CMI sont présentées dans le tableau 1 (page 290). Les résultats indiquent que le groupe avec des incidents disciplinaires $(\mathrm{N}=359)$ est plus jeune que le groupe sans incident $(\mathrm{N}=575)$, avec une moyenne de 32,7 ans comparativement à 38,2 ans $(\mathrm{t}=7,35, \mathrm{p}<0,01)$. En ce qui a trait aux différentes échelles de la section 1 du LS/CMI, le groupe avec des incidents obtient des résultats plus élevés à l'ensemble des échelles. Son résultat total à la section 1 est de 26,8 , considéré comme un niveau de risque élevé, un score significativement plus élevé $(t=-11,39, p<0,001)$ que celui des délinquants n'ayant pas fait l'objet de tels rapports.

La distribution de nos deux groupes dans les différents niveaux de risque du LS/CMI indique que $84,9 \%$ des membres du groupe ayant participé à des incidents disciplinaires se retrouvent avec un niveau de risque jugé élevé ou très élevé. Ce niveau est significativement plus élevé 


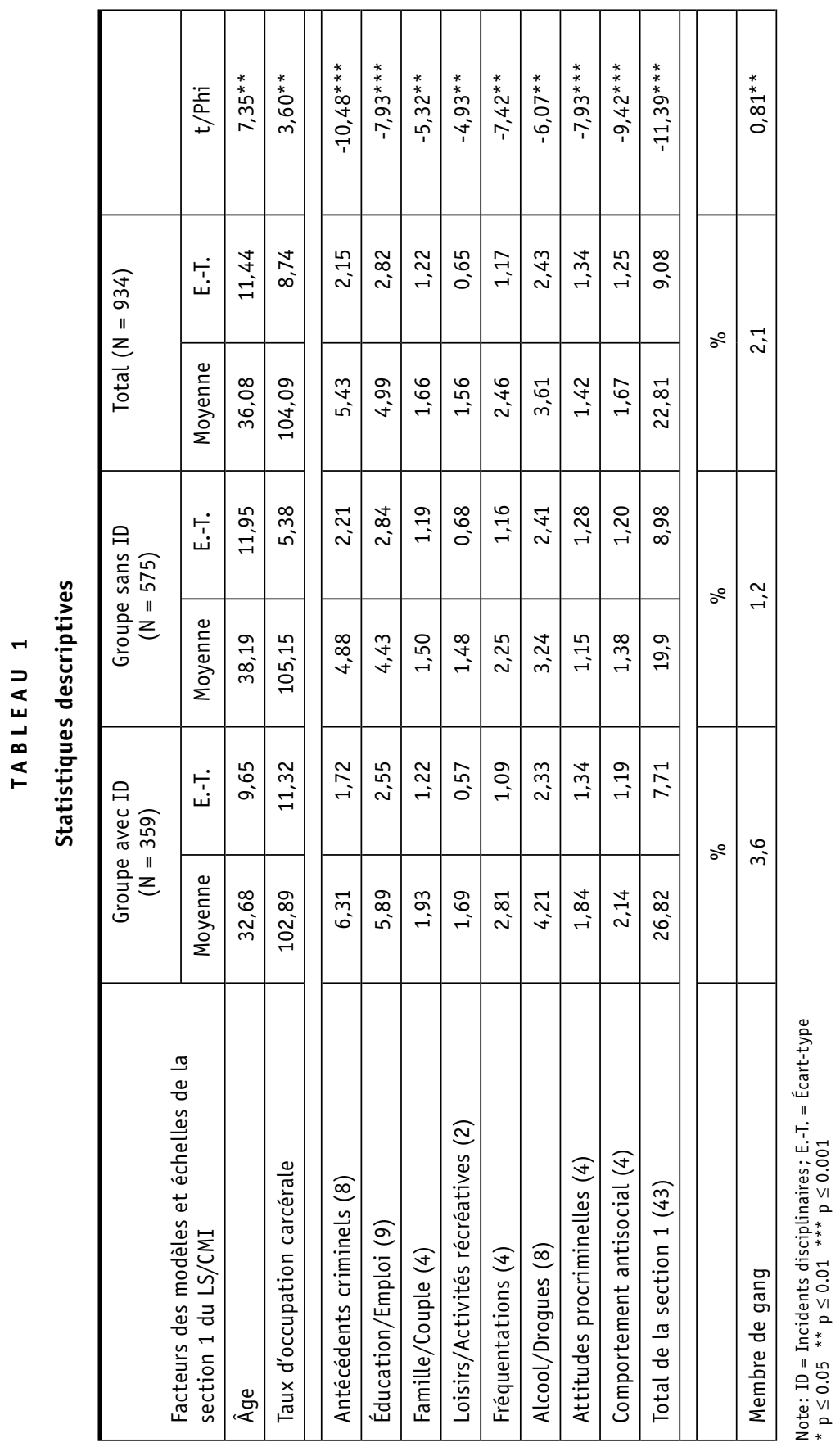


que pour les délinquants n'ayant pas fait l'objet de rapports d'incidents disciplinaires où $53 \%$ se retrouvent avec un niveau de risque comparable $\left(\chi^{2}=111,3, \mathrm{p}<0,001\right)$.

\section{Analyses d'aire sous la courbe ROC du LS/CMI}

Le tableau 4 (page 294) présente le résultat des analyses de l'aire sous la courbe (AUC) ROC pour les échelles de la section $1 \mathrm{du}$ LS/ CMI par rapport aux différents types d'incidents disciplinaires. L'étude des coefficients d'aire sous la courbe ROC donne une indication de la qualité générale de la prédiction. Elle offre donc l'occasion de connaître à quel point l'analyse permet de distinguer les vrais négatifs des vrais positifs. Cependant, dans une logique de gestion au quotidien, l'identification des vrais positifs a une valeur toute particulière. Puisque le coefficient AUC ne nous renseigne pas uniquement sur notre capacité à reconnaître correctement les auteurs d'incidents disciplinaires, le tableau 2 ci-dessous s'attarde à l'indice de proportion de prédiction positive (PPP) et à la sensibilité de chacun des modèles.

TABLEAU 2

AUC des échelles de la section 1 du LS/CMI par rapport aux différents types d'incidents disciplinaires (ID)

\begin{tabular}{|l|l|l|l|l|}
\hline $\begin{array}{l}\text { Échelles de la section 1 } \\
\text { du LS/CMI }\end{array}$ & $\begin{array}{l}\text { Violence } \\
\text { physique } \\
\text { AUC (ES) }\end{array}$ & $\begin{array}{l}\text { Violence } \\
\text { verbale } \\
\text { AUC (ES) }\end{array}$ & $\begin{array}{l}\text { Non } \\
\text { violent } \\
\text { AUC (ES) }\end{array}$ & $\begin{array}{l}\text { Total } \\
\text { ID } \\
\text { AUC (ES) }\end{array}$ \\
\hline Antécédents criminels (8) & $0,68(0,03)$ & $0,71(0,04)$ & $0,71(0,02)$ & $0,70(0,02)$ \\
\hline Éducation/Emploi (9) & $0,66(0,03)$ & $0,68(0,03)$ & $0,68(0,02)$ & $0,65(0,02)$ \\
\hline Famille/Couple (4) & $0,59(0,03)$ & $0,60(0,03)$ & $0,64(0,03)$ & $0,60(0,02)$ \\
\hline Loisirs/Activités récréatives (2) & $0,59(0,03)$ & $0,57(0,03)$ & $0,59(0,02)$ & $0,58(0,02)$ \\
\hline Fréquentations (4) & $0,59(0,03)$ & $0,64(0,03)$ & $0,65(0,02)$ & $0,64(0,02)$ \\
\hline Alcool/Drogues (8) & $0,64(0,03)$ & $0,62(0,03)$ & $0,64(0,03)$ & $0,62(0,02)$ \\
\hline Attitudes procriminelles (4) & $0,59(0,03)$ & $0,67(0,03)$ & $0,63(0,03)$ & $0,65(0,02)$ \\
\hline Comportement antisocial (4) & $0,68(0,03)$ & $0,71(0,03)$ & $0,73(0,02)$ & $0,67(0,02)$ \\
\hline Total de la section 1 (43) & $0,71(0,03)$ & $0,73(0,02)$ & $0,74(0,02)$ & $0,71(0,02)$ \\
\hline
\end{tabular}

Note: ID = Incidents disciplinaires; $E S=$ Erreur standard. 


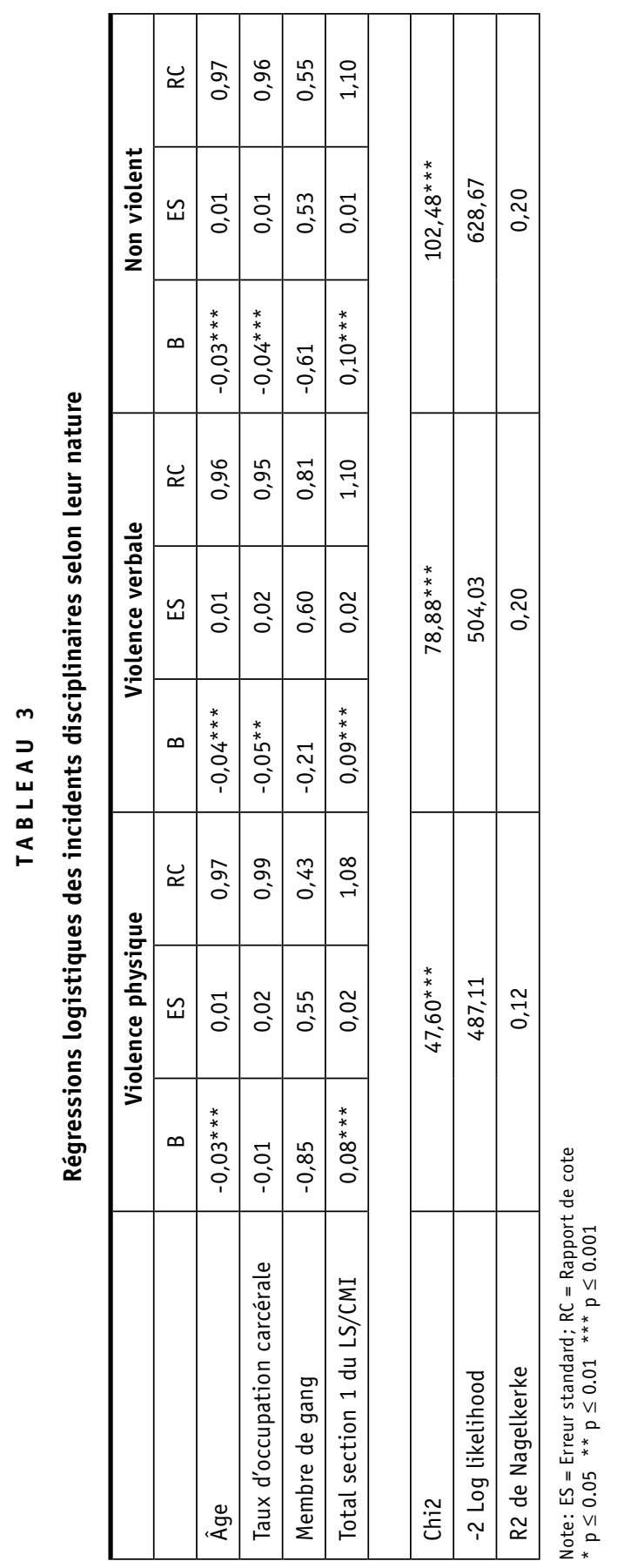


Les résultats d'analyses d'aire sous la courbe ROC sont significatifs à $\mathrm{p}<0,01$ pour l'ensemble des échelles du LS/CMI, et ce, indépendamment de la nature de l'incident. Pour le total de la section $1 \mathrm{du}$ LS/ CMI, on obtient une aire sous la courbe ROC supérieure à 0,70 pour les trois types d'incidents disciplinaires, soit de $0,71(p<0,001)$ pour les incidents de violence physique, de $0,73(\mathrm{p}<0,001)$ pour les incidents de violence verbale et de $0,74(p<0,001)$ pour les incidents non violents. De la même façon, l'indice AUC du total de cet instrument pour l'ensemble des incidents est de 0,71 ( $p<0,001)$, une validité prédictive par ailleurs qualifiée de modeste (Swets, 1988).

En ce qui a trait aux différentes échelles de la section 1, seuls les antécédents criminels et le comportement antisocial franchissent la barre des 0,70 pour la prédiction des différents types d'incidents. Le comportement antisocial semble prédire modestement les incidents disciplinaires de violence verbale (AUC $=0,71, \mathrm{p}<0,001)$ et les incidents non violents (AUC $=0,73, p<0,001$ ). Les antécédents criminels obtiennent un indice AUC supérieur à 0,70 pour les incidents de violence verbale (AUC $=0,71, \mathrm{p}<0,001$ ), les incidents non violents (AUC $=0,71, \mathrm{p}<0,001)$ et le total des incidents $(\mathrm{AUC}=0,70, \mathrm{p}<0,001)$. Aucune échelle du LS/CMI ne semble prédire de manière adéquate les incidents de violence physique à l'exception du total de la section 1 .

\section{Régression logistique des incidents disciplinaires selon leur nature et leur cible}

Cette section vise à présenter la validité prédictive du LS/CMI et des différentes variables contrôles. Les deux tableaux suivants présentent les résultats des modèles d'analyse de régression logistique pour les incidents disciplinaires selon leur nature (incidents de violence verbale, incidents non violents) et leur cible (incidents visant les membres du personnel ou un codétenu). Les indices de tolérance des analyses de régression ne présentent aucun problème de multicolinéarité.

Le tableau 3 (page 292) présente les résultats des régressions logistiques pour le total du LS/CMI en lien avec les incidents selon leur nature. Dans l'ensemble, le modèle prédit significativement les trois types d'incidents. L'indice R2 de Nagelkerke suggère que les incidents disciplinaires de violence verbale et non violents sont expliqués par une proportion de variance plus élevée $(\mathrm{R} 2=20,0 \%)$ que les incidents de violence physique $(\mathrm{R} 2=12,2 \%)$. 
De manière spécifique, les incidents de violence physique sont prédits significativement par l'âge $(\mathrm{RC}=0,97, \mathrm{p}<0,001)$ et le total au LS/CMI ( $R C=1,08, p<0,001)$. Plus le détenu est jeune et plus il est jugé à risque selon le LS/CMI, alors plus grande est la probabilité qu'il soit impliqué dans un incident de violence physique. Pour les incidents de violence verbale, trois facteurs sur quatre sont significatifs, soit l'âge $(\mathrm{RC}=0,96, \mathrm{p}<0,001)$, le taux d'occupation carcérale $(\mathrm{RC}=0,95$, $\mathrm{p}<0,01)$ et le total de la section $1 \mathrm{du}$ LS/CMI $(\mathrm{RC}=1,10, \mathrm{p}<0,001)$. Donc, plus le détenu est jeune avec un niveau de risque élevé au LS/ CMI et plus le taux d'occupation carcérale est faible, plus il risque d'être impliqué dans un incident de violence verbale faisant l'objet d'un rapport. En ce qui a trait aux incidents non violents, les résultats sont similaires aux incidents de violence verbale. L'âge $(\mathrm{RC}=0,97$, $\mathrm{p}<0,001)$, le taux d'occupation carcérale $(\mathrm{RC}=0,96, \mathrm{p}<0,001)$ et le total au LS/CMI $(\mathrm{RC}=1,10, \mathrm{p}<0,001)$ prédisent significativement ce type d'incidents.

Le tableau suivant présente les résultats des régressions logistiques pour les incidents disciplinaires selon leur cible, ou selon les personnes impliquées.

TAB LEA U 4

Régressions logistiques des incidents disciplinaires selon la cible

\begin{tabular}{|c|c|c|c|c|c|c|}
\hline & \multicolumn{3}{|c|}{$\begin{array}{c}\text { Incidents impliquant un } \\
\text { autre détenu }\end{array}$} & \multicolumn{3}{|c|}{$\begin{array}{c}\text { Incidents impliquant un } \\
\text { intervenant }\end{array}$} \\
\hline & B & ES & $\mathrm{RC}$ & B & ES & $\mathrm{RC}$ \\
\hline Âge & $-0,04^{* * *}$ & 0,01 & 0,97 & $-0,04^{* * *}$ & 0,01 & 0,96 \\
\hline $\begin{array}{l}\text { Taux d'occupation } \\
\text { carcérale }\end{array}$ & $-0,03^{* *}$ & 0,01 & 0,97 & $-0,05^{* * *}$ & 0,01 & 0,95 \\
\hline Membre de gang & $-0,56$ & 0,49 & 0,57 & $-1,06^{*}$ & 0,50 & 0,35 \\
\hline Total Section 1 du LS/CMI & $0,09 * * *$ & 0,01 & 1,09 & $0,08^{* * *}$ & 0,01 & 1,08 \\
\hline Chi2 & \multicolumn{3}{|c|}{$140,07^{* * *}$} & \multicolumn{3}{|c|}{$88,96^{* * *}$} \\
\hline-2 Log likelihood & \multicolumn{3}{|c|}{882,18} & \multicolumn{3}{|c|}{564,19} \\
\hline R2 de Nagelkerke & \multicolumn{3}{|c|}{0,23} & \multicolumn{3}{|c|}{0,19} \\
\hline
\end{tabular}

Note: $\mathrm{ES}=$ Erreur standard; $\mathrm{RC}=$ Rapport de cote

${ }^{*} \mathrm{p} \leq 0.05^{* *} \mathrm{p} \leq 0.01{ }^{* * *} \mathrm{p} \leq 0.001$ 
Le tableau 4 présente les résultats des régressions logistiques pour le total du LS/CMI en lien avec les incidents selon leur cible. Dans l'ensemble, le modèle prédit significativement les deux types d'incidents. L'indice R2 de Nagelkerke suggère que les incidents impliquant un autre détenu sont expliqués par une proportion de variance plus élevée $(\mathrm{R} 2=22,8 \%)$ que les incidents impliquant un intervenant $(\mathrm{R} 2=$ $19,4 \%)$.

Plus spécifiquement, les incidents impliquant un autre détenu sont prédits significativement par l'âge ( $\mathrm{RC}=0,97, \mathrm{p}<0,001)$, le taux d'occupation carcérale $(\mathrm{RC}=0,97, \mathrm{p}<0,01)$ et le total au LS/CMI $(\mathrm{RC}=1,09, \mathrm{p}<0,001)$. Plus le détenu est jeune avec un niveau de risque élevé au LS/CMI et plus le taux d'occupation carcérale est faible, plus la probabilité est élevée qu'il soit impliqué dans ce type d'incidents. En ce qui a trait aux incidents impliquant un intervenant, l'âge $(\mathrm{RC}=$ $0,97, p<0,001)$, le taux d'occupation carcérale $(\mathrm{RC}=0,96, \mathrm{p}<0,001)$, l'affiliation à un gang $(\mathrm{RC}=0,35, \mathrm{p}<0,05)$ et le total au LS/CMI $(\mathrm{RC}$ $=1,10, \mathrm{p}<0,001)$ prédisent significativement ce type d'incidents.

\section{Discussion}

L'effet du taux d'occupation carcérale et de l'appartenance à un gang

L'un des objectifs de cette étude était de tester la contribution de facteurs tels que le taux d'occupation carcérale et l'appartenance à un gang dans la prédiction des incidents disciplinaires. Les résultats suggèrent une relation négative entre le taux d'occupation carcérale et les incidents disciplinaires, et ce, indépendamment de leur sévérité et de leur cible. De tels résultats contrastent par ailleurs avec les travaux précédents (Megargee, 1976; Gaes et McGuire, 1985; Gendreau et al., 1997; Wooldredge et al., 2001; Reisig, 2002) indiquant une augmentation des probabilités d'être impliqué dans un incident disciplinaire en contexte de surpopulation. Pourtant, certains chercheurs observent également une telle diminution des incidents disciplinaires en contexte de surpopulation (Gaes et McGuire, 1985; Tartaro, 2002). Une des hypothèses formulées est que le sens de cette relation serait imputable aux comportements des agents de services correctionnels. En situation de surpopulation, les agents de services correctionnels auraient moins l'occasion de relever les incidents disciplinaires. Ces incidents sont 
davantage sujets à de simples avertissements qu'à des rapports disciplinaires complets. En d'autres termes, la surpopulation modifierait sensiblement la réponse des agents de services correctionnels, se traduisant par une augmentation du chiffre noir et une diminution du caractère systématique de la consignation de tels événements (Ruback et Carr, 1993). Cependant, il est possible de croire que le niveau d'événements rapportés resterait inchangé pour les incidents plus sérieux, comme les incidents de violence physique. Les résultats de la présente étude indiquent que la surpopulation ne semble pas influencer les incidents disciplinaires de violence physique $(\mathrm{RC}=0,99, \mathrm{p}>0,05)$. Cette influence moindre de la surpopulation sur ce type d'incidents est congruente avec notre hypothèse, mais ne permet pas de la confirmer pour autant. Il reste que ce phénomène peut s'observer également dans la criminalité hors murs. Le taux d'événements rapportés est toujours plus grand pour les crimes graves en raison de la difficulté à dissimuler ce type de crimes et ses fâcheuses répercussions. Pour Ellis (1984), les fluctuations dans la réponse du personnel devraient être davantage prises en considération dans l'observation des effets de la surpopulation sur les incidents disciplinaires.

Pour sa part, l'affiliation à un gang donne lieu à des résultats qui divergent des études précédentes. La plupart des études constatent que cette affiliation en milieu carcéral augmente la probabilité de commettre des incidents disciplinaires violents (Ralph et Marquart, 1991; Shelden, 1991; Gaes et al., 2002; DeLisi et al., 2004; Griffin et Hepburn, 2006; Trulson, 2007). Nos résultats semblent indiquer l'effet inverse. Les membres de gangs font moins fréquemment l'objet de rapports d'incidents disciplinaires impliquant un intervenant que les non-membres. Le sens inattendu de cette relation peut dépendre de plusieurs raisons d'ordre méthodologique, comme un manque de critères fidèles quant à la mesure du phénomène (Guay, 2008). Par ailleurs, au Québec, vers le début des années 2000, des agents de services correctionnels avaient été la cible d'attaques pendant un long conflit que se livraient des bandes rivales de motards criminalisés. Encore aujourd'hui, de nombreux actes d'intimidation sont commis par les membres de gangs de rue à l'endroit des agents correctionnels (Boutet, 2008). Il est donc possible que la consignation des incidents impliquant des membres de gangs soit moins grande par peur de représailles. Finalement, la plupart des établissements de détention du Québec regroupent les membres de gangs selon leur affiliation pour une gestion 
du risque plus efficace. Il est possible qu'un tel choix ait eu comme conséquence de diminuer les confrontations entre les membres de gangs rivaux, ou tout simplement qu'il ait fait en sorte que les agents correctionnels optent pour une intervention réduite lors d'altercations entre membres de gangs.

\section{La validité prédictive du LS/CMI}

L'objectif principal de la présente étude était de tester la validité prédictive du LS/CMI en lien avec les différents types d'incidents disciplinaires. Une lecture attentive des résultats des différentes échelles de la section $1 \mathrm{du}$ LS/CMI en lien avec les incidents disciplinaires permet de constater une prédiction modeste (AUC $>0,70$ ) des échelles relatives aux antécédents criminels et aux comportements antisociaux. Ces résultats sont appuyés par plusieurs études qui indiquent généralement une relation significative entre la gravité de la carrière criminelle et la probabilité de faire l'objet de rapports d'incidents disciplinaires (Flanagan, 1983 ; Goetting et Howsen, 1986; Cooper et Werner, 1990; Gendreau et al., 1997; Woolredge et al., 2001 ; DeLisi et Munoz, 2003; Winfree et al., 1994; Cunningham et Sorensen, 2007; Trulson, 2007). Les criminels de carrière, particulièrement ceux qui manifestent des attitudes antisociales, font plus fréquemment l'objet de tels rapports.

Les travaux de Kroner et Mills (2001) et de Gendreau et ses collaborateurs (1997) indiquaient déjà que le LSI-R, l'ancêtre du LS/CMI, semblait offrir une avenue prometteuse dans la prédiction des incidents disciplinaires. Les résultats de cet article permettent de confirmer l'efficacité du LS/CMI dans la prédiction des incidents disciplinaires. À ce sujet, les performances du LS/CMI sont comparables aux résultats du Risk Assessment Scale for Prison (Cunningham et al., 2005), un instrument spécifiquement conçu pour évaluer le risque sécuritaire. Le RASP et le LS/CMI obtiennent tous deux des indices d'aire sous la courbe ROC de 0,71 pour la prédiction des incidents disciplinaires violents. Bien que les résultats soient identiques, les outils génériques offrent des avantages importants. Un instrument de gestion du risque tel que le LS/CMI, en plus de prédire le risque d'incident disciplinaire et la récidive, offre des balises importantes en ce qui concerne les principales cibles d'intervention. De plus, puisque les outils génériques font partie de l'évaluation de la clientèle délinquante, nul besoin de traduire ni de valider un nouvel instrument pour ensuite former l'ensemble des 
agents de probation à la cotation d'un nouvel outil. À la lumière de ces avantages et des résultats de la présente étude, l'utilisation du LS/CMI se présente dorénavant comme une solution plus qu'avantageuse dans l'évaluation du risque sécuritaire.

La principale limite de cette étude est le caractère non significatif de l'effet du facteur de l'affiliation à un gang dans nos modèles de régression logistique. Seulement 20 détenus sont identifiés comme faisant partie d'un gang dans l'ensemble de notre échantillon de 934 détenus. Ce faible niveau de base peut en partie expliquer les performances modestes de ce facteur dans notre modèle. Également, en raison de l'impact significatif du taux d'occupation carcérale sur l'occurrence des incidents disciplinaires, des facteurs supplémentaires tels que le niveau de sécurité des différents secteurs, le lieu et le moment de l'incident ont déjà prouvé leur contribution (McCorkle, Miethe et Drass, 1995; Gendreau et al., 1997 ; Jiang et Fisher-Giorlando, 2002). Ces facteurs auraient potentiellement permis de bonifier le modèle.

\section{Références}

Andrews, D. A., \& Bonta, J. (1995). The Level of Service Inventory-Revised. Toronto: Multi-Healt Systems.

Andrews, D. A., \& Bonta, J. (2006). Psychology of criminal conduct (4e éd.). Newark (NJ): Anderson Publishing Co.

Andrews, D. A., Bonta, J., \& Wormith, J. S. (2004). Manual for the Level of Service/Case Management Inventory (LS/CMI). Toronto, Canada: Multi-Health Systems.

Andrews, D. A., Dowden, C., \& Rettinger, J. L. (2001). Special populations within Canada. In J. A. Winterdyck (Ed.), Corrections in Canada: Social reactions to crime (170-212). Toronto, Ontario: Prentice Hall.

Assemblée nationale du Québec (2009). L'état des services correctionnels au Québec. Journal des débats de la commission des institutions, 41 (15).

Bonta, J. (1989). Native inmates: Institutional responses, risks, and needs. Canadian Journal of Criminology, 31, 49-62.

Bonta, J., \& Motiuk, L. L. (1987). The diversion of incarcerated offenders to correctional halfway houses. Journal of Research in Crime and Delinquency, 24, 302-323.

Boutet, M. (2008). L'intimidation dans le parcours criminel des gangs de rue. Séminaire Gangs et Délinquance, Montréal, Canada.

Butcher, J. N., Dahlstrom, W. G., Graham, J. R., Tellegen, A., \& Kaemmer, B. (1989). Minnesota Multiphasic Personality Inventory (MMPI-2): Manual for administration and scoring. Minneapolis: University of Minnesota Press. 
Cao, L., Zhao, J., \&Van Dine, S. (1997). Prison disciplinary tickets: A test of the deprivation and importation models. Journal of Criminal Justice, 25, 103113.

Cooper, R. P., \& Werner, P. D. (1990). Predicting violence in newly admitted inmates: A lens model analysis of staff decision making. Criminal Justice and Behavior, 17, 431-447.

Coulson, G., Ilacqua, G., Nutbrown, V., Giulekas, D., \& Cudjoe, F. (1996). Predictive utility of the LSI for incarcerated female offenders. Criminal Justice and Behavior, 23, 427-439.

Cunningham, M. D., \& Sorensen, J. R. (2006). Actuarial models for assessing prison violence risk: Revisions and extensions of the risk assessment scale for prison (RASP). Assessment, 13, 253-265.

Cunningham, M. D., \& Sorensen, J. R. (2007). Predictive factors for violent misconduct in close custody. The Prison Journal, 87, 241-253.

Cunningham, M., Sorensen, J., \& Reidy, T. (2005). An actuarial model for assessment of prison violence risk among maximum security inmates. Assessment, 12, 40-49.

DeLisi, M., \& Munoz, E. A. (2003). Future dangerousness revisited. Criminal Justice Policy Review, 14, 287-305.

DeLisi, M., Berg, M. T., \& Hochstetler, A. (2004). Gang members, career criminals and prison violence: further specification of the importation model of inmate behaviour. Criminal Justice Studies, 17, 369-383.

Dilulio, J. (1991). No escape: The future of American corrections. New York: Basic Books.

Edens, J. F., Poythress, N. G., \& Lilienfeld, S. O. (1999). Identifying inmates at risk for disciplinary infractions: A comparison of two measures of psychopathy. Behavioral Sciences and the Law, 17, 435-443.

Ellis, D. (1984). Crowding and prison violence. Criminal Justice and Behavior, 11 (3), 277-308.

Endrass, J., Rossegger, A., Urbaniok, F., Laubacher, A., \& Vetter, S. (Sous presse). Predicting violent infractions in a Swiss state penitentiary: a replication study of the PCL-R in a population of sex and violent offenders.

Flanagan, T. J. (1983). Correlates of institutional misconduct among state prisoners. Criminology, 21, 29-39.

Gaes, G. G., \& McGuire, J. W. (1985). Prison violence: The contribution of crowding versus other determinants of prison assault rates. Journal of Research in Crime and Delinquency, 22, 41-65.

Gaes, G. G., Wallace, S., Gilman, E., Klein-Saffran, J., \& Suppa, S. (2002). The influence of prison gang affiliation on violence and other prison misconduct. The Prison Journal, 82 (3), 359-385.

Gendreau, P., Goggin, C., \& Law, M. (1997). Predicting prison misconducts. Criminal Justice and Behavior, 24, 414-431.

Gendreau, P., Goggin, C., \& Smith, P. (2002). Is the PCL-R really the "unparalleled" measure of offender risk? A lesson in knowledge cumulation. Criminal Justice and Behavior, 29, 397-426. 
Girard, L. (1999). The Level of Supervision Inventory - Ontario Revision: Risk/need assessment and recidivisms. Thèse de doctorat inédite, University of Ottawa, Ottawa, Ontario.

Girard, L., \& Wormith, J. S. (2004). The Predictive Validity of the Level of Service Inventory-Ontario Revision on General and Violent Recidivism among Various Offender Groups. Criminal Justice and Behavior, 31, 150-181.

Goetting, A., \& Howsen, R. M. (1986). Correlates of prisoner misconduct. Journal of Quantitative Criminology, 2, 49-67.

Guay, J. P. (2008). Mesurer les gangs et l'appartenance aux gangs. Séminaire Gangs et Délinquance, Montréal, Canada.

Griffin, M. L., \& Hepburn, J. R. (2006). The effect of gang affiliation on violent misconduct among inmates during the early years of confinement. Criminal Justice and Behavior, 33, 419-466.

Hanson, R. K., \& Thornton, D. (1999). Static-99: Improving actuarial risk assessments for sex offenders (User Report No. 99-02). Ottawa, Canada : Department of the Solicitor General of Canada.

Hare, R. D. (2003). The Hare Psychopatby Checklist-Revised. Toronto: MultiHealth Systems.

Harris, G. T., Rice, M. E., \& Quinsey, V. L. (1993). Violent Recidivism of Mentally Disordered Offenders: The Developement of a Statistical Prediction Intrument. Criminal Justice and Behavior, 20, 315-335.

Hochstetler, A., \& DeLisi, M. (2005). Importation, deprivation, and varieties of serving time: An integrated-lifestyle-exposure model of prison offending. Journal of Criminal Justice, 33, 257-266.

Hoge, R. D., Andrews, D. A., \& Leschied, A.W. (1995). The Risk/Need Inventory. The Ministry of Community and Social Services. Toronto, Ontario.

Hollin, C. V., \& Palmer, E. M. (2003). The Level of Service Inventory-Revised profile of English prisoners: Risk and reconviction analysis. Criminal Justice and Behavior, 33, 347-366.

Jiang, S., \& Fisher-Giorlando, M. (2002). Inmate misconduct: A test of the deprivation, importation, and situational models. The Prison Journal, 82, 335-358.

Kroner, D., \& Mills, J. (2001). The accuracy of five risk appraisal instruments in predicting institutional misconduct and new convictions. Criminal Justice and Behavior, 28, 471-489.

Landreville, P., \& Charest, M. (2004). Analyse prospective de la population des établissements de détention du Québec: Rapport final. Montréal: Centre international de criminologie comparée.

Lovell, D., \& Jemelka, R. (1996). When inmates misbehave: The costs of discipline. The Prison Journal, 76, 165-179.

Loza, W., \& Dhaliwal, G. (1997). Psychometric evaluation of the Risk Appraisal Guide (RAG): A tool for assessing violent recidivism. Journal of Interpersonal Violence, 12, 779-793.

Mackenzie, D. L. (1987). Age and adjustment to prison: Interactions with attitudes and anxiety. Criminal Justice and Behavior, 14, 427-447. 
McCorkle, R. C., Miethe, T. D., \& Drass, K. A. (1995). The roots of prison violence: A test of deprivation, management, and 'not-so total' institution models. Crime and Delinquency, 41, 317-331.

Megargee, E. I. (1976). Population density and disruptive behaviour in a prison setting. In A. K. Cohen, G. F. Cole \& R. G. Bailey (Eds.), Prison Violence (135-144). Lexington, MA: Heath.

Ministère de la Sécurité publique du Québec (2005). Plan stratégique 2005-2008. Québec: Gouvernement du Québec.

Nowicka-Sroga, M. (2003). The Level of Service Inventory - Ontario Revision and phase II, young offenders. Canadian Psychology, 44 (2a), 21.

Ouimet, M. (1993). Surveiller qui? Une étude des infractions disciplinaires commises par les personnes incarcérées. Forum, Recherche sur l'actualité correctionnelle, 5 (2), 40.

O’Donnell, I., \& Edgar, K. (1998). Routine victimisation in prisons. The Howard Journal, 37 (3), 266-279.

Paterline, B. A., \& Petersen, D. M. (1999). Structural and social psychological determinants of prisonization. Journal of Criminal Justice, 27, 427-441.

Ralph, P. H., \& Marquart, J. W. (1991). Gang violence in Texas prisons. The Prison Journal, 71, 38-49.

Reisig, M. (2002). Administrative control and inmate homicide. Homicide Studies, 6, 84-103.

Rettinger, J. (1998). A recidivism follow-up study to investigate risk and need within a sample of provincially sentenced women. Thèse de doctorat, Carleton University, Ottawa, Ontario.

Rice, M. E., \& Harris, G. T. (1995). Violent recidivism: Assessing predictive validity. Journal of Consulting and Clinical Psychology, 63, 737-748.

Robinson, D., \& Mirabelli, L. (1996). Rapport de synthèse. Résumé des constatations du Sondage national auprès des détenus. Numéro $B-14$, Canada: Division de la recherche et développement correctionnels, Services correctionnels du Canada.

Ruback, R. B., \& Carr, T. S. (1993). Prison crowding over time; The relationship of density and changes in density ti infractions rates. Criminal Justice and Behavior, 20, 130-148.

Serin, R. C. (1991). Psychopathy and violence in criminals. Journal of Interpersonal Violence, 6, 423-431.

Service correctionnel du Canada (2007). Rapport sur le rendement. Canada: Service correctionnel du Canada.

Shelden, R. G. (1991). A comparison of gang members and non-gang members in a prison setting. The Prison Journal, 71, 50-60.

Shields, I. W. (1993). The use of the Young Offender-Level of Service Inventory (YO-LSI) with adolescents. IARCA Journal, 5, 10-26.

Shields, I. W., \& Simourd, D. J. (1991). Predicting predatory behavior in a population of incarcerated young offenders. Criminal Justice and Behavior, 18, 180-194. 
Sjostedt, G., \& Grann, M. (2002) Risk assessment: What is being predicted by actuarial prediction instrument? International journal of forensic mental bealth, 1 (2), 179-183.

Sparring, M. (1982). The Youth Service LSI. Thèse de doctorat inédite, Departement de Psychologie, Carleton University, Ottawa.

Swets, J. A. (1988). Measuring the accuracy of diagnostic systems, Science, 240 (4857), 1285-1293.

Tartaro, C. (2002). The impact of density on jail violence. Journal of Criminal Justice, 30, 499-510.

Toch, H. (1977). Living in prison: The ecology of survival. New York: The Free Press.

Thomas, C. W., \& Foster, S. C. (1973). The importation model perspective on imate social roles: An empirical test. The Sociological Quaterly, 14, 226-234.

Trulson, C. R. (2007). Determinants of disruption: Institutional misconduct among State-Committed delinquents. Youth Violence and Juvenile Justice, 5, 7-34.

Van Voorhis, P. (1994). Measuring prison disciplinary problems: A multiple indicators approach to understanding prison adjustment. Justice Quarterly, 11, 679-709.

Walters, G. D. (2003). Predicting institutional adjustment and recidivism with the Psychopathy Checklist factor scores: A meta-analysis. Law and Human Behavior, 27, 541-558.

Walters, G. D., Duncan, S. A., \& Geyer, M. D. (2003). Predicting disciplinary adjustment in inmates undergoing forensic evaluation: A direct comparison of the PCL-R and PAI. Journal of Forensic Psychiatry and Psychology, 14, 382393.

Walters, G. D., White, T. W., \& Denney, D. (1991). The Lifestyle Criminality Screening Form: Preliminary data. Criminal Justice and Behavior, 18, 406-418.

Webster, C. D., Eaves, D., Douglas, K., \& Wintrup, A. (1995). The HCR-20 Scheme: The assessment of dangerousness and risk. Vancouver: Simon Fraser University and Forensic Psychiatric Services Commission of British Columbia, Simon Fraser University Press.

Winfree, L. T., Mays, G. L., Crowley, J. E., \& Peat, B. J. (1994). Drug history and prisonization: Toward understanding variations in inmate institutional adaptations. International Journal of Offender Therapy and Comparative Criminology, 38, 281-296.

Wooldredge, J. D. (1991). Correlates of deviant behavior among inmates of U.S. correctional facilities. Journal of Crime and Justice, 14, 1-25.

Wooldredge, J. D., Griffin, T., \& Pratt, T. C. (2001). The relevance of hierarchical models for empirical study of inmate behavior. Justice Quarterly, 18, 901-929.

Wright, K. N. (1985). Developing the Prison Environment Inventory. Journal of Research in Crime and Delinquency, 22, 257-77.

Wright, K. N. (1991). A study of individual, environmental, and interactive effects in explaining adjustment to prison. Justice Quarterly, 8, 217-242. 
Young, M. H., Justice, J. V., \& Erdberg, P. (2004). Assault in prison and assault in prison psychiatric treatment. Journal of Forensic Sciences, 29, 1-9.

Zamble, E. (1992). Behavior and adaptation in long-term prison inmates: Descriptive longitudinal results. Criminal Justice and Behavior, 19, 409-425.

\begin{abstract}
The purpose of this study was to address the critical need to improve accuracy in risk assessment for prison misconducts and hence improve decision-making. With the increased prison crowding in the correctional facilities in Quebec, there is a critical need for the development of better instruments to assist clinicians in their task. Since February 2007, all offenders sentenced for a violent crime or to six month or more are now evaluated with the French-Canadian version of the LS/CMI. Although the LS/ CMI is generally good at predicting recidivism, it was not specifically designed to predict prison misconducts. The present study investigates the potential predictive validity of the LS/CMI. The sample consisted of 934 male offenders sentenced for a violent crime or to 6 month or more in the Correctional Services of Quebec between February 2007 and December 2007. In general, results indicate that the LS/CMI can predict violent and non violent misconducts.
\end{abstract}

KEYWORDS - Prison, misconducts, risk assessment, actuarial.

RESUMEN - En su plan estratégico, el ministerio de Seguridad pública de Quebec destaca los problemas actuales de sobrepoblación en las cárceles de Quebec. Dicho fenómeno corre el riesgo de contribuir al aumento de conflictos entre los detenidos $y$, por extensión, a la emergencia de incidentes disciplinarios. En ese contexto, se vuelve necesario mejorar la gestión de riesgos asociados a ese tipo de incidentes. El objetivo principal del presente estudio fue poner a prueba la validez predictiva del LS/CMI sobre ocurrencia de incidentes disciplinarios. La muestra consistió en 934 detenidos encarcelados en el Servicio correccional de Quebec (SCQ) entre enero y diciembre de 2007. Durante ese periodo, todos los detenidos fueron evaluados en las semanas que siguieron a su admisión con la versión canadiense del LS/CMI. Los resultados permiten confirmar la eficacia del LS/CMI en la predicción de incidentes disciplinarios independientemente de su naturaleza.

PLABRAS CLAVE - Incidentes disciplinarios, prisión, gestión de riesgo, herramienta actuarial. 